\title{
La tragedia greca per bambini
}

\author{
The Greek tragedy for children
}

\author{
Alberto REGAGLIOLO ${ }^{1}$ \\ Uniwersytet Kardynała Stefana Wyszyńskiego w Warszawie
}

\begin{abstract}
I bambini dagli otto / nove anni non hanno ancora una completa e chiara idea sulla morte; nonostante ciò, un modo per avvicinarli a riflettere su questo tema sensibile, può essere attraverso la letteratura, in particolare con la tragedia greca. Presentare la tragedia greca già alle elementari avvicina inevitabilmente al tema dell'educazione delle morte e del lutto oltre ad affrontare la questione della sofferenza. Occorre comunque individuare all'interno del repertorio classico di Sofocle, Euripide e Eschilo alcuni paladini positivi che possano guidare i bambini nella comprensione di alcuni momenti tragici adattati. Le istituzioni, i docenti e le associazioni sono fondamentali nell'accompagnare i bambini in questo percorso alla scoperta della morte e del lutto attraverso il curriculum scolastico e ad altre attività alternative. Alcune possibilità didattiche rientrano nell'educazione multisensoriale, esperienziale e ludica. Qui si presenta una proposta analizzando alcuni elementi della tragedia dell'Antigone da mettere in pratica alle elementari.

Keywords: Tragedia greca, Death studies, Educazione primaria
\end{abstract}

\begin{abstract}
1 https://orcid.org/0000-0002-5766-0064

Uniwersytet Kardynała Stefana Wyszyńskiego w Warszawie,

Instytut Filologii Klasycznej i Kulturoznawstwa

a.regagliolo@uksw.edu.pl
\end{abstract}

Children at eight / nine years old do not have yet a complete and clear idea of the death; nevertheless, one way to approach them to reflect on this sensitive issue can be through literature, in particular with the Greek tragedy. Introducing the Greek tragedy already in primary school inevitably brings the child closer to the theme of the education of death and loss as well as addresses the issue of suffering. However, it is necessary to identify within the classical repertoire of Sophocles, Euripides and Aeschylus some positive characters who can 
guide children in understanding some adapted tragic moments. Institutions, teachers and associations are essential in accompanying children on this journey to discover death and mourning through the school curriculum and other alternative activities. Some teaching possibilities fall within multisensory, experiential and playful education. Here a proposal is presented by analyzing some elements of the Antigone tragedy to be put into practice in primary school.

Keywords: Greek tragedy, Death studies, Primary Education

\section{Introduzione}

La ricezione degli elementi della cultura classica, campo di studio molto ampio e al tempo stesso proficuo, ci permette di analizzare in dettaglio, approfondire e soprattutto riflettere sulla società odierna in relazione con il passato. Questo ci consente di conoscere e comprendere come un determinato elemento, sia esso un'opera letteraria, un mito, un pensiero filosofico, sia cambiato, ma al tempo stesso come si relazioni con l'epoca contemporanea, con le nuove ideologie, con il nuovo contesto storico, nell'intento di ricoprire una nuova identità al passo con l'ordine temporale.

Un genere che offre un interessante e più che mai aperto ambito di studio è la tragedia greca che ha avuto una forte ripercussione nel mondo contemporaneo (Molinari, 1977) (Bosch, 1979) (Ragué Arias, 1986) (López \& Pociña, 2010) (Azcue, 2009) (Duroux \& Urdician, 2010) (Mee \& Foley, 2011) e che è stata rappresentata in molti paesi, in diverse lingue e con caratteristiche alquanto differenti. La tragedia greca, oltre a farci riflettere sul cambio passato e presente e sugli elementi della ricezione dei classici, come lo era nel passato, può essere anche un genere educativo con una propria funzione pedagogica. Affrontare la tragedia greca già in tenera età, però, non è un compito semplice perché gli argomenti trattati non sono di facile comprensione per i bambini ma anche perché soprattutto vi sono elementi tragici ed eventi che oggigiorno, come allora, sono considerati estremi e non sempre segnali positivi. Affrontare determinati elementi della tragedia greca può essere comunque uno stimolo positivo per trattare temi quali la morte, il lutto, il dolore e la giustizia, tra gli altri.

Uno spunto positivo per introdurre la tragedia greca già alle elementari è l'Antigone di Sofocle. L'Antigone, è stata oggetto di studio e di interesse di diversi autori come Jean Rotrou, Racine, Hegel, Kierkegaard, mentre nel Novecento ricordiamo Heidegger, Bertolt Brecht, Jean Anouilh, Janusz Głowacki, María Zambrano, Miguel del Arco, solo per menzionarne alcuni. 
L'Antigone oltre a presentare alcuni elementi particolari di una paladina della giustizia, è una figura positiva che si scontra contro lo Stato per amore del fratello morto Polinice a cui è stata vietata la sepoltura.

In questo studio si ripercorrerà la peculiarità della tragedia greca analizzando il concetto di tragedia e di morte, per poi presentare delle attività didattiche relazionate con la tragedia classica dell'Antigone per poter avvicinare i bambini a temi ancora poco conosciuti, come la morte e il lutto.

\section{La tragedia. Il tragico e la morte}

Parlare di tragedia ci rimanda alla tradizione classica greca per eccellenza. Il teatro nell'antica Grecia era composto da due generi ben specifici: la commedia e la tragedia greca per l'appunto. Quest' ultima, nata tra il V e il IV sec a. C, veniva celebrata nei mesi di marzo e aprile durante le festività religiosa in onore al dio Dioniso. In questa occasione ogni tragediografo doveva rappresentare tre opere e un dramma satiresco, che poi venivano votati da una giuria. 'La tragedia era la rappresentazione scenica mediante attori (prima due, poi tre) di un'azione (drama) attraverso la recitazione, la musica, la danza. Al prologo iniziale (recitato da uno o più personaggi) seguiva una párodo (canto d'ingresso del coro) e una serie di episodi separati tra loro da stásimi (canti del coro). La sezione conclusiva era l'ésodo o "uscita». Il coro, che rappresentava i cittadini della polis, dialogava coi personaggi per bocca del corifèo' (Piazzi, 2010: 25). La tragedia però non è solo legata alla cultura greca classica, bensì anche alla letteratura successiva. In effetti, quello che accomuna tanto le tragedie classiche quanto le tragedie moderne e contemporanee è l'elemento del tragico, requisito sine qua non si può parlare di tragedia. Il tragico, infatti, indipendentemente dagli elementi culturali, può essere definito in modo qualitativo, come lo propone Cesarino: 'definire il tragico è forse possibile dicendo cosa non è. Non è il quotidiano, il banale, il normale. È, quindi, lo straordinario, l'eccezionale' (Casarino, 2010: 13). I dizionari propongono diverse definizioni di tragedia. Come la definisce il dizionario Garzanti, è un 'avvenimento tragico, luttuoso' ${ }^{2}$, mentre per l'Oxford Dictionary è 'a very sad event or situation, especially one that involves death's $\mathrm{o}$ ancora 'suffering with a sad end'4. A sua volta, il Dizionario Merriam la definisce come 'a serious drama typically describing a conflict between the protagonist and a superior

\footnotetext{
${ }^{2}$ www.garzantilinguistica.it/ricerca/?q=tragedia

${ }^{3}$ www.oxfordlearnersdictionaries.com/definition/english/tragedy

${ }^{4}$ https://dictionary.cambridge.org/dictionary/english/tragedy
} 
force (such as destiny) and having a sorrowful or disastrous conclusion that elicits pity or terror's. La RAE la descrive come una 'situación o suceso luctuoso y lamentable que afecta a personas o sociedades humanas'6, mentre invece il Dizionario Larousse la definisce come un 'Événement funeste, terrible'7. Infine nel Priberam troviamo: 'cena ou acontecimento triste, grave ou

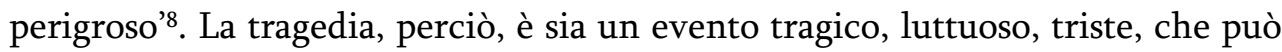
includere la morte, la sofferenza, il terrore, una triste, catastrofica o pericolosa conclusione, sia un conflitto tra più forze che incidono e influenzano l'uomo o la società umana.

Parte della tragedia, come si è visto, è anche il tema della morte e del lutto, un argomento a cui l'adulto avrà fatto fronte almeno una volta nella propria vita, sia direttamente che indirettamente. Il significato di morte, tuttavia, non è semplice da capire specialmente in età infantile; esso rimane tuttavia un concetto di indispensabile comprensione per la vita dell'essere umano. La mancanza di una consapevolezza della morte e del lutto può generare conseguenze di notevole importanza sul piano psicologico e sociale dell'individuo, sia esso adulto o infante (Krepia, Krepia \& Tsilingiri, 2017). Le ripercussioni negative, infatti, arrivano specialmente quando la famiglia non parla della morte o non permette al bambino di assistere al funerale o di fare domande in merito al tema. Secondo Silverman et al. (1995) il bambino in questo caso avrà difficoltà a capire e a formulare una propria idea della morte e non saprà gestire le proprie emozioni, diversamente da chi ha dei genitori più aperti e disponibili al dialogo, favorevoli a coinvolgere il proprio figlio nell'elaborazione del lutto. In questo ultimo caso il bambino avrà la possibilità di maturare il concetto di morte. Secondo alcuni studi non parlare della morte sarebbe un gesto di protezione da parte della società nei confronti dei più piccoli (Schoen, Burgoyne \& Schoen, 2004), per questo molte volte viene trattata in modo silenzioso (Fonseca \& Testoni, 2011: 164 citato in McGloughlin, 2017: 52) D'altra parte è anche vero che la comprensione del concetto di morte dipende anche dallo stadio cognitivo del bambino (Hunter \& Smith, 2008): essendo nella fase preoperatoria (2-6 anni, seguendo la suddivisione piagetiana) è ancora difficile comprendere il tema nella sua totalità, come ad esempio il concetto di irreversabilità. La morte, infatti, è diversa da quella che un bambino piccolo vede nei cartoni animati, dove

\footnotetext{
${ }^{5}$ www.merriam-webster.com/dictionary/tragedy

${ }^{6} \mathrm{https} / / /$ dle.rae.es/tragedia

${ }^{7}$ www.larousse.fr/dictionnaires/francais/trag\%C3\%A9die/78925

${ }^{8}$ https://dicionario.priberam.org/trag\%C3\%A9dia
} 
il protagonista deceduto torna poi magicamente in vita (Schonfeld \& Quackenbush, 2009: 32). La morte è un evento irreversibile il cui concetto si comprende al meglio quando la perdita subita è quella di una persona cara anziché quella di una figura estranea o lontana (Orbach, Weiner, Har-Even \& Eshel 1994-1995).

Avvicinarsi alla comprensione della morte è un compito arduo, tuttavia è fondamentale che la scuola e la famiglia, oltre che le diverse istituzioni, siano in grado di aiutare i bambini e i più piccini nel rapportarsi con la morte e il lutto (Baker, Sedney \& Gross, 1992) per poter permettere di formare dei cittadini responsabili che possano vivere nella società.

\section{La tragedia nel curriculum elementare}

La Death Education (DeEd), disciplina inclusa tra i Death Studies, si propone come obiettivo quello di educare alla morte, ovvero dare quegli strumenti per poter comprendere un passaggio obbligatorio a cui tutti prima o poi andiamo incontro. L'educazione passa attraverso diversi mezzi; vi sono diversi progetti che propongono delle esperienze educative per avvicinare il bambino alla morte e al lutto, come per esempio il progetto Uno Sguardo al Cielo. Percorso di avvicinamento all'Elaborazione del Lutto ${ }^{9}$ arrivato all'VIII edizione e svoltosi nella provincia di Ferrara. I progetti e le attività in questo ambito sono davvero molteplici e sorprende come una disciplina così 'sensibile' abbia piano piano iniziato a prendere piede nelle scuole elementari.

Oltre alle diverse associazioni e attività di supporto dei vari centri, inserire delle attività didattiche-educative all'interno del percorso scolastico sembra essere un'esigenza più che mai necessaria a cui dovranno far fronte i docenti ${ }^{10}$. Oltre ad avere degli ausili esterni, lo stesso curriculum educativo

\footnotetext{
9 Il progetto è sotto la guida di Paola Bastianoni (Università degli Studi di Ferrara) con la collaborazione delle agenzie di onoranze funibri: AMSEF e PAZZI (www.unosguardoalcielo.com/ progetto).

${ }^{10}$ Molte volte però sono gli stessi docenti ad aver problemi non sapendo come affrontare il tema. Emerge, di conseguenza, la necessità di una maggior preparazione nell'ambito della docenza, specialmente nei futuri docenti che si sentono ancora persi e non formati nel parlare della morte sia in ambito generale ma anche in quelli più specifici in età infantile (Galande, 2015). Un aiuto concreto, ad esempio, è quello offerto dall'Associazione internazionale Rainbows cui missione è quella di fornire gratuitamente supporto a tutti i giovani che fanno fronte 'alla perdida di una persona, alla morte, al divorzio / separazione, alla deportazione, all'incarcerazione o ad altri traumi (www.rainbows.org) e che mette a disposizione esperti che collaborano con le scuole
} 
può offrire un supporto ai bambini. L'idea di inserire nel curriculum l'Educazione alla morte si pone come elemento fondamentale per la crescita e lo sviluppo del bambino (King-McKenzie, 2011). Diverse sono le materie presenti nel ciclo primario che possono fornire un nesso al tema; tra gli esempi annotiamo la biologia, in particular modo in merito al funzionamento dell'organismo e gli argomenti ad esso attinenti (malattie, crescita, invecchiamento); le scienze sociali e le lingue per quanto riguarda la conoscenza delle tradizioni dei diversi paesi, basti pensare al día de los muertos, festa celebrata in Messico il due di novembre per onorare i morti. La storia, inoltre può essere un punto di partenza con gli Egizi, gli Etruschi e le loro città dei morti. Anche la religione, qualora presente nel curriculum, può essere una materia utile per avvicinarsi al rito funebre nelle diverse religioni. Oltre a ciò, la letteratura presenta vari, interessanti e validi spunti per poter riflettere. Vi sono diversi generi che si legano al tema della morte, come la biografia, il mito, il diario (si pensi ad Anna Frank e al libro adattato e reso fumetto per bambini e ragazzi ${ }^{11}$ ), ma anche la poesia. Un genere poco affrontato però è la tragedia, molto probabilmente per la crudeltà a volte utilizzata tra le righe, basti solo ricordare Medea che lasciata da Giasone, il marito, uccide i due figli e la nuova moglie. Seppur esitando per il dolore che comunque le causerà, Medea attuerà il macabro finale per vendicarsi della nuova vita del marito. Questo tragico evento, sebbene la cronaca di ogni giorno sia piena di tristi finali, a volte è difficile da digerire anche per un adulto. All'interno della vita scolastica dei bambini, il tragico si trova frequentemente nel mito, nelle leggende e nelle opere di Omero (Iliade e Odissea) e in quella di Virgilio (Eneide); rimangono generalmente assenti invece i tragediografi greci: Sofocle, Euripide ed Eschilo. La tragedia greca fornisce tuttavia un percorso educativo, a nostro avviso, fondamentale per le nuove generazioni che oggi più che mai sono assorte nel loro mondo virtuale e gamificato in cui si immedesimano nel ladro di turno o nello spietato agente segreto che senza scrupoli uccide chi si mette d'intralcio, non riuscendo poi a riflettere sulla morte tramite altri mezzi e modi. Già nel passato la tragedia greca era di grande utilità perché aveva un compito pedagogico verso il popolo, verso la collettività. Effettivamente ancora oggi 'le opere classiche non hanno perso la loro rilevanza, ma costituiscono punti chiave da cui considerare i problemi legati

e centri, fornendo anche training a docenti per poter supportare il loro lavoro quotidiano in ambito scolastico.

${ }^{11}$ A. FRANK, D. POLONSKY, Anne Frank's Diary: The Graphic Adaptation, Penguin, 2018. 
alla razionalizzazione della cultura [...], ma anche legati all'istruzione ${ }^{12}$ (Grinberg, 2006: 142). Avvicinarsi alla tragedia greca non significa perció solo avvicinarsi alla cultura del passato, ma anche alla realtà dei giorni nostri; nonostante siano passati migliaia di anni e nonostante la cultura greca antica e quella nostra contemporanea siano diverse, in realtà le dinamiche umane sono le stesse. Per questo la tragedia greca è così contemporanea: l'essere umano è sempre geloso, triste, ferito, vendicativo, iracondo, irrazionale, ecc ${ }^{13}$. Essa è un elemento precipuo per porgersi dubbi e domande applicando gli stessi interrogativi che si applicano al mito o alla leggenda, perché possono essere dei «potenti stimoli per l'immaginazione, specialmente quando coinvolgono il soprannaturale $o$ il fantastico; e possono sensibilizzare $i$ bambini sulle domande di base sulla moralità e sulla verità: "E corretto aver ucciso il mostro?", "E successo davvero?", "Potrebbe essere successo così.»14 (Department for Education and Science, 1988: 10). Questi sono elementi che diventano anche degli spunti e dei punti di partenza per affrontare alcuni temi rilevanti, come la dicotomia tra i valori eroici/valori democratici, la religiosita/razionalità, il diritto divino/diritto umano, la responsabilità collettiva/responsabilità individuale, la volontà divina/volontà individuale e il destino/libertà. Benché questi molte volte includano concetti e pensieri astratti, possono essere un inizio per porre delle importanti basi per sviluppare il pensiero critico e la riflessione.

\section{Antigone}

Se si prende in esame il caso di Antigone, si può osservare come anche la tragedia possa offrire un incipit interessante per aprire nuove riflessioni

\footnotetext{
12 Traduzione dell'autore.

${ }^{13}$ Uno studio recente (Olechowska, 2015) ci mostra, infatti, l'utilizzo della tragedia classica come mezzo idiologico durante il periodo comunista in Polonia. Nella realtà spagnola ne è un esempio l'Antigone di José María Pemán che rivendica il partito franchista proprio perché, in quel determinato periodo storico e dal punto di vista del partito di Franco, c'era la volontà e sicuramente la necessità di far emergere anche nell'arte del teatro questa supremazia del potere verso il popolo. Da questi esempi si può osservare come l'opera teatrale (scritta e interpretata) sia molte volte la riflessione di un determinato periodo. Questo significa che, sebbene nei diversi paesi si rappresentino sempre più tragedie e commedie di stampo greco e romano, l'influenza del hic et nunc - ovvero lo specifico contesto e il fattore temporale - delineano una condizione di variabile che, attraverso l'insieme dell'atto recitato, può far emergere una risposta di cambio (dunque di ricezione diversa) in una specifica società.

14 Traduzione dell'autore.
} 
anche in età infantile. Attraverso le dinamiche della scrittura di Sofocle, la protagonista, Antigone, rappresenta una spaccatura della democrazia durante il V sec. B.C. Essa apre un eterno dibattito in relazione alla legge sacra e profana e una legge assoluta, non scritta dagli dei, a cui fa appello la protagonista, lei 'misura della legge nella tragedia di Sofocle' (Mancini, 2014: 10) - che manifesta una coscienza morale che dovrebbe condurre ad una modifica dell'editto emanato da Creonte, sovrano di Tebe. Il tema, anche se di radice antica, offre degli elementi moderni: la figura della donna, la realtà femminile che si scontra contro lo Stato, contro il potere. Ella lo fa per una ragione ber precisa: dare degna sepoltura al fratello morto, Polinice. Una figura, quella di Antigone, che prende realtà e coscienza della propria posizione nella società e gestisce in prima persona le proprie azioni imbattendosi in una ribellione che è, come sottolinea la critica teatrale della versione di Del Arco, 'la enseña visible de un personaje eterno precisamente porque afronta dicotomías capitales: la felicidad o el duelo, la honra a los muertos o el apego a los vivos, la felicidad o la angustia, la obediencia a la Ley o la lealtad a uno mismo, el yo o el Estado'15. Ed è proprio il dolore, la degna sepoltura e il lutto, oltre alla giustizia, che diventano dei validi elementi per poter avvicinare i bambini alla tragedia greca. Antigone è un simbolo positivo nonostante lo scenario intorno a lei. Un personaggio che opera in nome della giustizia e dell'amore per il fratello morto e anche se parte di una tragedia, Antigone diventa comunque un modello positivo. Senza cadere nella banalità, Antigone è una paladina della giustizia che affronta una situazione complicata e critica per una buona causa, tema per eccellenza raccontato e affrontato anche nelle fiabe, oltre che nei molteplici film della Disney o della Pixar. Pensiamo ad esempio a Elsa ed Anna, le protagoniste di Frozen I e II, cartone animato adattato dalla fiaba 'The Snow Queen' di Hans Christian Andersen, che si scontrano contro il male. Anche loro affrontano il lutto oltre ad andare incontro a diversi pericoli e a situazioni estreme e pericolose. Nonostante non tutti i protagonisti delle tragedie greche possano diventare o essere dei modelli positivi, Antigone lo è e diventa pertanto una connessione essenziale per presentare la tragedia greca già alle elementari. Antigone, oltre ad offrire un modello positivo, ci permette di comprendere diversi meccanismi psicologici e di trattare più da vicino il tema della morte e della sepoltura. Avvicinare i bambini alla figura dell'Antigone è perciò motivo di comprensione del dolore, della giustizia e del lutto stesso per iniziare a comprendere l'assenza della persona amata, l'irreversibilità, l'elaborazione del lutto e il ricordo.

${ }^{15}$ www.teatroateatro.com/criticas/critica-de-antigona/12899/ 
La comprensione della tragedia dell'Antigone è fondamentale per poter far riflettere sulla morte e lavorare sull'educazione alla morte. Gli eventi drammatici originali per comprendere la tragedia dell'Antigone sono: 1) la morte di Eteocle e Polinice che nei Sette contro Tebe si uccidono tra di loro, 2) l'opposizione della sorella Ismene, 3) il decreto di Creonte che non permette la sepoltura di Polinice, 4) la punizione di Antigone per aver cercato di dare sepoltura al fratello, 5) il suicidio di Antigone, 6) il suicidio del promesso sposo di Antigone, Emone, 7) Il suicidio della moglie di Creonte, Euridice, 8) la disperazione di Creonte. Non tutti però sono necessari per presentare ed introdurre l'Antigone e per affrontare insieme ai bambini alcuni elementi della tragedia greca. Già con i punti 1 (la morte di Polinice), 3 (il decreto di Creonte), 4 (il castigo) si ha una struttura solida per presentare e continuare comunque con una parte della drammaticità del testo originale.

Se si ripercorrono questi tre punti, si possono notare alcuni elementi di rilevanza per introdurre la tragedia greca. In primis 1) la morte di Polinice non viene descritta nell'Antigone di Sofocle ma nei Sette contro Tebe di Eschilo. Essendo pertanto un fatto scontato e implicito nell'Antigone, riprendere la morte del fratello aiuta a far capire la drammaticità e la conseguenza degli eventi successivi. L'ideale è perciò adattare sì il testo al livello richiesto, ma anche poter fare delle aggiunte per far comprendere meglio la parte che altrimenti sarebbe mancante. La morte di Polinice è l'elemento scatenante degli altri eventi successivi: da una causa ci si trova di fronte ad una (o più) conseguenze. Polinice fornisce una chiave per comprendere la mancanza di una persona per poter discutere sul tema della morte e del lutto, dell'assenza e dell' amore per una persona.

Ad aumentare la drammaticità della tragedia dell'Antigone è il 2) decreto di Creonte. Esso ci mette in relazione con la giustizia. Cos'è giusto o sbagliato? Creonte vieta espressamente di poter seppellire Polinice. Anche la nostra società ci indica cosa una persona può fare o non fare e come si deve comportare: la legge e il rispetto della legge è un tema che viene affrontato ogni giorno non solo dall'adulto ma anche dal bambino. Già in tenera età il bambino impara piano piano a seguire degli ordini e a rispettare delle indicazioni date dagli altri per poter vivere 'correttamente' nella società. Non rispettare le regole significa infrangerle, disubbidire. Il tema della disubbidienza è comune nell'infanzia: il bambino, infatti, più volte avrà disubbidito a qualcuno anche per cose piccolo e banali. Deve essere chiaro per il piccolo pubblico che disubbidire può avere diversi livelli di conseguenza; infatti anche Cappuccetto Rosso disubbidisce alla mamma e si ferma a parlare con il lupo ma lo fa 
in buona fede. Ariel, la sirenetta, disubbidisce più volte al padre Tritone e si allontana, come anche Nemo. La disubbidienza, perciò, è un tema centrale anche nella fiaba. L'argomento viene descritto dettagliatamente nelle funzioni della fiaba dall'antropologo e linguista russo, Vladimir Propp, nel libro Morphology of the folktale. Delle 31 funzioni presentate particolarmente interessante per questo punto è la seconda funzione che riguarda il divieto del protagonista di compiere un'azione (1968: 26) e la terza, l'infrazione del divieto stesso (Ibid, 26). In molti cartoni animati, e normalmente nelle fiabe, disobbedire è un passo fondamentale per la storia e solitamente segue l'aspirazione e il cuore del protagonista in un preciso momento ${ }^{16}$. Per poter capire l'azione di Antigone, ovvero il tentativo di seppellire il fratello con della sabbia, è indispensabile perciò lavorare su più piani per comprendere perché Antigone abbia disobbedito ad una legge, perché, in fin dei conti, Antigone non vuole fare del male a nessuno. In merito a ciò, è bene ricordare che fino ai nove anni, indicativamente, un bambino non riesce ancora bene a dividere l'aspetto morale dalla legge (Weyers, 2014); è pur vero, però, che l'educazione e la scuola sono indispensabili per l'acquisizione delle norme sulla moralità, per imparare a relazionarsi all'interno della società già a partire dalle attività quotidiane. A questo punto, un adattamento su più piani è richiesto per giustificare Antigone e capire perciò che non è l'antagonista.

La sepoltura di Polinice è un altro nesso fondamentale. La morte di una persona ma anche di un animale corrisponde ad un rituale diverso da cultura a cultura; pensiamo ad esempio ai faraoni i quali venivano interrati in piramidi monumentali e il cui corpo doveva essere preservato per il concetto del dopo vita. La sepoltura di Polinice apre la possibilità di affrontare una varietà di argomenti di grande interesse come i costumi e le usanze dei riti funebri anche nei popoli antichi e che si possono presentare in diverse lezioni. Oltre ad analizzare un metodo particolare di inumazione, la morte di Polinice, tuttavia, ci permette di capire come dare degna sepoltura, ovvero una sepoltura meritevole e adeguata ad una persona, che può risultare ancora un concetto abbastanza astratto visto che molti bambini a questa età non hanno ancora assistito ad un funerale.

Antigone, dopo aver tentato di coprire con un po' di sabbia il fratello deceduto, viene castigata dallo zio Creote che la farà rinchiudere in una grotta. Il quarto punto, ovvero il castigo di Antigone pone l'interrogativo nuovamente sul giusto e il non giusto avvicinando il pubblico al tema dei diritti umani e della giustizia.

${ }^{16}$ https://therhetoricofdisney.weebly.com/disobedience.html\# 


\section{Proposte didattiche}

Per avvicinarsi alla tragedia di Sofocle, prima di tutto ci si deve occupare dell'adattamento del testo, fondamentale per proporre un testo adeguato sia dal punto di vista contenutistico e linguistico al lettore. Molte sono le forme per far comprendere e far riflettere i bambini sulla morte e sul lutto utilizzando la tragedia greca. Vi sono diverse attività che si possono proporre già alle elementari. In merito a ciò è necessario ricordare quanto sia fondamentale l'aspetto ludico, multisensoriale ed esperienziale per l'apprendimento e l'acquisizione di nuovi elementi, concetti e temi durante la tappa infantile.

Come attività da proporre alle elementari, indicativamente a partire dagli otto-nove anni, oltre alla lettura della tragedia (adattata), è possibile anche recitare dei versi, delle parti. Infatti, l'approcciarsi al testo molte volte non è abbastanza perché non si riescono a notare tutti gli aspetti importanti tra le righe (Vodickova, 2009: 150); per questo motivo si consiglia di mettere in pratica la tragedia come role-play (recitazione e danza) anche per mettere in gioco le emozioni (Usakli, 2018, Gazalka; Idogho, 2019) perché il teatro diventa, utilizzando le parole di Grotowski, 'un veicolo per lo studio di se stessi e per l'esplorazione di sê ${ }^{17}$ (Romanska, 2012: 12). Da non sottovalutare, dunque, la funzione artistica e manipolativa. L'arte, infatti, aiuta nel far fronte al lutto (Walter, 2012): oltre ad essere l'arte-terapia molto comune ha come obiettivo quello di 'consentire al paziente di cambiare e svilupparsi a livello personale $^{18}$ (Wood, 2011: 243). L'arte, intesa dunque nelle sue più ampie espressioni, lascia un'ulteriore libertà espressiva al bambino per riflettere, attraverso diversi strumenti, sulla morte. A questo proposito si può proporre: 1) la costruzione di uno scrigno dei ricordi. Gli oggetti hanno un valore che va al di là del costo e del tempo ma anche della qualità. L'oggetto infatti è usato per ricordare i propri cari, coloro che sono deceduti (Layne, 2000; Hallam \& Hockey, 2001) ma anche per ricordare la propria identità e provenienza (Marschall, 2019). Gli studenti possono creare uno scrigno inserendo degli oggetti relazionati ad una persona cara che non c'è più o immaginare quello che Antigone avrebbe potuto mettere dentro la scatola. Inoltre, i bambini si possono cimentare nella 2) costruzione di una statuetta per ricordare la persona che se ne è andata. Le statue commemorative nel passato ma anche oggigiorno sono un modo per rendere anche pubblica l'importanza della persona deceduta.

\footnotetext{
17 Traduzione dell'autore.

18 Traduzione dell'autore.
} 
Oltre all'arte, anche la scrittura è una forma di espressione utile per far fronte al lutto (Matthews, 2017; 2019). Per introdurre degli esercizi più da un punto di vista linguistico, oltre alla drammatizzazione di scene adattate e di lunghezza controllata, si può proporre 3) la redazione di una lettera indirizzata ad una persona che non c'è più o a Polinice. Inoltre si può anche scrivere 4) un decalogo di cose che può fare Antigone per cercare di essere più felice (es: andare alla tomba del fratello, fare una passeggiata, andare a trovare gli amici...).

Per lavorare ancora con le emozioni, i bambini possono costruire 5) un barattolo delle emozioni, un barattolo in cui si possono inserire diversi aggettivi riguardanti le emozioni di Antigone nell'aver perso il fratello. Qui, oltre a riflettere sulle emozioni, si lavora anche sulla ricerca degli aggettivi.

Altresì i bambini possono utilizzare anche la storia e l'ordine cronologico creando 6) un albero genealogico della loro famiglia scrivendo il nome, la parentela e l'anno. Inoltre, visto che nei paesi più occidentali è ancora frequente l'inumazione della salma, un'attività interessante è relazionata al giardinaggio. Scavare una piccola buca per poter 7) seminare e poi far crescere alcune piantine può rappresentare un modo diverso di studiare il ciclo della vita.

Ultimo, ma non meno importante, è quello di dare la possibilità ai bambini di creare una connessione con il passato imparando come gli antichi greci in epoca classica preparavano il funerale delle persone care. Si possono così conoscere gli indumenti utilizzati, le decorazioni che si preparavano, i luoghi in cui il tutto veniva predisposto. Questo sarà utile per mettere in scena e dunque ricordare, vivere in prima persona, un evento drammatico seppur drammatizzato, mettendo in pratica il learning by doing freobeliano, imparando attraverso l'esperienza.

\section{Conclusioni}

La scuola e i docenti, insieme alle varie associazioni, sono fondamentali per poter creare un collegamento per introdurre degli elementi relazionati all'educazione alla morte. Diverse sono le materie che il curriculum di studio offre per avvicinare i più piccoli al tema della morte e del lutto, come le scienze, la religione, le lingue, ma anche la letteratura. Essa, in effetti, tra i suoi diversi generi include anche la tragedia. La letteratura classica antica è un infinito pozzo di saperi, di elementi da cui oggigiorno continuiamo ad attingere dipingendo il nostro presente con i colori e le tecniche del passato. 
La tragedia greca è uno di questi colori più attivi e che molte volte fornisce una chiave di lettura e di interpretezione del nostro presente ma che svolge anche una funzione pedagogica. Avvicinarsi alla tragedia greca classica, specialmente alle elementari, non è un compito semplice, soprattutto perché il tragico richiede l'utilizzo di elementi a volte di difficile comprensione. Proporre un percorso educativo alle elementari coinvolgendo i bambini alla scoperta della tragedia greca (adattata), è comunque un mezzo per comprendere e conoscere piano piano, in modo graduale, la morte, il lutto, l'irreversibilità, la mancanza della persona amata e il dolore. Sebbene la tragedia greca sia per antonomasia il genere più tragico, esso può diventare comunque a sua volta un testo pedagogico avvicinando i bambini alla scoperta dei personaggi, delle loro avventure, ma anche delle loro sofferenze. In fin dei conti non siamo poi così lontani dalle fiabe e dai cartoni Disney in cui da una situazione di mancanza iniziale si scatena una serie di eventi che portano finalmente alla risoluzione e al finale '...e vissero felice e contenti'. Certo, una differenza è sicuramente il finale. Però vi sono elementi che riconducono tuttavia alla fiaba, come l'allontanamento, l'abbandono, il lutto, la sofferenza, il superamento delle prove, il castigo, l'infrazione delle norme, solo per citarne alcuni. Una chiave per proporre la tragedia greca e potersi avvicinare alla comprensione e alla riflessione sul tema della morte è proporre dei personaggi che, seppur all'interno di un contesto tragico, riescono comunque ad emergere per il loro carattere positivo e per le loro azioni nella ricerca della giustizia. E Antigone è a tutti gli effetti una paladina greca classica della giustizia. Presentare una tragedia greca non è un compito semplice, prima di tutto perché non tutte le tragedie si dispongono a presentare dei modelli positivi. Effettivamente, presentare una madre come Medea che uccide i propri figli sembra alquanto crudele; nuovamente, comunque, non siamo però lontani dalle tante fiabe classiche in cui la matrigna tenta il figlicidio come in Biancaneve o in Hans e Gretel. Nonostante ciò, la selezione del testo e l'adattamento sono fondamentali per procedere con l'introduzione di una tragedia. Il testo dell'Antigone propone vari elementi su cui lavorare in classe come la morte di Polinice, il decreto di Creonte, la sepoltura o la sfida di Antigone. Tenendo in considerazione questi elementi è possibile lavorare su più piani proponendo attività di riflessione tramite la drammatizzazione, lavorando perciò anche sulle emozioni, sull'uso delle parole con la recitazione; ma anche attraverso attività manipolative come la costruzione di oggetti specifici, come lo scrigno dei ricordi o una statuetta commemorativa. Altresì, si può anche introdurre la scrittura di una lettera o anche la costruzione di un albero genealogico. 
Anche se ne è solo un esempio, Antigone può diventare un nesso per presentare la tragedia greca ed essere parte integrante dell'educazione alla morte, perché la morte possa diventare, con il tempo, un momento significativo e non un evento scontato e di difficile comprensione nell'età adulta.

\section{BIBLIOGRAFIA}

Anouilh, J. (1988). Antigone; Reclam Philipp Editor.

Azcue, V. (2009). Antígona en el teatro español contemporáneo, Acotaciones, pp. 33-46.

Baker, J. E. \& Sedney, M. A. \& Gross, E. (1992). Psychological tasks for bereaved children. American Journal of Orthopsychiatry, 62, 1992, pp. 105-116.

Bosh, M. C. (1979). Antígona en la literatura moderna, Barcelona, Universidad de Barcelona, Secretariado de Publicaciones.

Brecht, B. (2000). Antigone, L'Arche éditeur.

Casarino, S. (2010). Il senso del tragico e la tragedia, Atti del convegno Dala Ghislieri, Aracne Editrice.

Department of Education and Science (1998). Classics from 5-16. Curriculum Matters, 12, London HMSO.

Duroux, R. \& Urdician, S. (2010). Les Antigones contemporaines: de 1945 à nos jours, ClermontFerrand, Presses Universitaires Blaise Pascal, Collection 'Mythographies et sociétés'.

Fonseca, L. M. \& Testoni, I. (2011). The emergence of Thanatology and current practice in Death Education, Omega Journal of Death and Dying, 64(2), 2011, pp. 157-169.

Frank, A. \& Polonsky, D. (2018). Anne Frank's Diary: The Graphic Adaptation, Penguin.

Galande, N. (2015). Death and Its Didactics in Pre-School and Primary School, Procedia, Social and Behavioral Sciences 185, 2015, pp. 91-97.

Grinberg, S. M. (2006). Education and the Tragedy of Culture: Pedagogical Notes on the Rationalization of Knowledge, Philosophy of Education, pp. 142-149.

Hallam, E. \& Hockey, J. (2001). Death, Memory and Material Culture. Berg, Oxford.

Hunter, S. B. \& Smith, D. E. (2008). Predictors of children's understandings of death: age, cognitive ability, death experience and maternal communicative competence, Omega, 57 (2), 2008, pp. 143-162.

Idogho, J. A. (2019). Teaching Emotional Intelligence through Drama: A Report of the UNIBEN Consultancy Primary II Pupils Project, International Journal of Caring Sciences; September-December, Vol. 10, Issue 3, 2019, pp. 1717-1721.

King-McKenzie, E. (2011). Death and Dying in the Curriculum of Public Schools: Is there a place? Journal of Emerging Knowledge on Emerging Markets, Vol. 3.

Krepia, M., Krepia, V. \& Tsilingiri, M. (2017). School Children's Perception of the Concept of Death.

Layne, L. (2000). He was a real baby with real baby things' A Material Culture Analysis of Personhood, Parenthood and Pregnancy Loss, Journal of Material Culture, Vol. 5(3), 2000, pp. 321-345.

López, A. \& Pociña, A. (2010). La eterna pervivencia de Antígona; Flor. 11., 21, pp. 345-370.

Mancini, M. (2014). Nomos e Polis fra l'Antigone e il Critone Momenti del tragico nel mondo antico, Edizioni ETS. 
Marshall, S. (2019). Memory objects': Material objects and memories of home in the context of intra-African mobility, Journal of Material Culture, pp. 1-17.

Matthews, A. (2017). Writing through the Pain: An Autoethnographic Exploration of Grief, the Doctoral Process, Dissertation Difficulties, and Doctoral Attrition; The University of Toledo.

Matthews, A. (2019). Writing through grief: Using auto-ethnography to help process grief after the death of a loved one; Methodological Innovations September-December, 2019, pp. 1-10.

Mcgloughlin, C. (2017). The challenges that face the primary school teacher in coping with grief and loss in a multicultural setting. MA Thesis, University of Limerick.

Mee, E. B. \& Foley, H. P. (2011). Antigone on the Contemporary World Stage, Oxford University Press.

Molinari, C. (1977). Storia di Antigone (De Sofocle al Living Theatre). Un mito nel teatro occidentale, Bari, De Donato.

Olechowska, E. (2015). Classical Antiquity on Communist Stage in Poland. Ancient Theatre as an Ideologicall Medium: A Critical Review, Faculty of Artes Liberales, University of Warsaw.

Orbach, I., Weiner, M., Har-Even, D. \& Eshel, Y. (1994-1995). Children's perception of death and interpersonal closeness to the dead person, Omega: Journal of Death and Dying, 30(1), pp. $1-12$.

Piazzi, F. (2010). Autori, testi e percorsi, Cappelli Editore.

Propp, V. (1968). Morphology of the folktale, American Folklore Society Bibliographical and Special Series, University of Texas Press Austin.

Ragué Arias, M. J. (1986). Los Personajes Femeninos de la Tragedia Griega en el Teatro Español del siglo $X X$, Tesis de Doctorado, Barcelona.

Romanska, M. (2012). The Post-traumatic Theatre of Grotowski and Kantor: History and Holocaust in 'Akropolis' and 'Dead Class' (Anthem Studies in Theatre and Performance), Anthem Press.

Schoen, A. A. \& Burgoyne, M. \& Schoen, S. (2004). Are the developmental needs of children in America adequately addressed in the grieving process? Journal of Instructional Psychology, 31(2), pp. 143-150.

Schonfeld, D. J. \& Quackenbush, M. (2009). After a Loved One Dies. How Children Grieve And how parents and other adults can support them; New York Life Foundation.

Silverman, P. R., Weiner, A. \& Elad, N. (1995). Parent-child communication in bereaved Israeli families. Omega, 31, pp. 275-293.

Speece, M. W. \& Brent, S. B. (1984). Children's understanding of death: A review of three components of a death concept. Child Development, 55, pp. 1671-1686.

Usakli, H. (2018). Drama Based Social Emotional Learning. Global Research in Higher Education, Vol. 1, n. 1.

Vodickova, M. (2009). Interpretation of poetry through drama activities, Journal of NELTA, 14(12), pp. 146-15.

Walter, T. (2012). How People Who Are Dying or Mourning Engage with the Arts. Music and Arts in Action, Vol. 4 n. 1.

Weyers, S. (2014). Wie verstehen Kinder und Jugendliche das Recht? Sechs Phasen der Entwicklung rechtlichen Denkens [How do children and adolescents understand the law? Six stages of legal thinking development]. In C. Kölbl, G. Mey (Eds.), Gesellschaftsverständnis: Entwicklungspsychologische Perspektiven [Social 
understanding: Developmental perspectives], Giessen and Lahn, Germany: Psychosozial Verlag, pp. 105-131.

Wood, M. (2011). The contribution of art therapy to palliative medicine. In Hanks G., Cherny N. I., Christakis N. A. Fallon M., Kaasa S., Portenoy R. K., Oxford Textbook of Palliative Medicine; Oxford University Press.

\author{
Sitografia \\ https://dicionario.priberam.org/trag\%C3\%A9dia (9/4/2020) \\ https://dictionary.cambridge.org/dictionary/english/tragedy (9/4/2020) \\ https://dle.rae.es/tragedia (9/4/2020) \\ https://rainbows.org/why-teachers-need-rainbows (9/4/2020) \\ www.garzantilinguistica.it/ricerca/?q=tragedia $(9 / 4 / 2020)$ \\ www.larousse.fr/dictionnaires/francais/trag\%C3\%A9die/78925 (9/4/2020) \\ www.merriam-webster.com/dictionary/tragedy (9/4/2020) \\ www.oxfordlearnersdictionaries.com/definition/english/tragedy $(9 / 4 / 2020)$ \\ www.rainbows.org $(9 / 4 / 2020)$ \\ www.unosguardoalcielo.com/progetto (9/4/2020)
}

